\title{
Time Series Classification with Discrete Wavelet Transformed Data: Insights from an Empirical Study
}

\author{
Daoyuan Li Tegawendé F. Bissyandé Jacques Klein Yves Le Traon \\ University of Luxembourg \\ \{daoyuan.li, tegawende.bissyande, jacques.klein, yves.letraon\}@uni.lu
}

\begin{abstract}
Time series mining has become essential for extracting knowledge from the abundant data that flows out from many application domains. To overcome storage and processing challenges in time series mining, compression techniques are being used. In this paper, we investigate the loss/gain of performance of time series classification approaches when fed with lossy-compressed data. This empirical study is essential for reassuring practitioners, but also for providing more insights on how compression techniques can even be effective in reducing noise in time series data. From a knowledge engineering perspective, we show that time series may be compressed by $90 \%$ using discrete wavelet transforms and still achieve remarkable classification accuracy, and that residual details left by popular wavelet compression techniques can sometimes even help achieve higher classification accuracy than the raw time series data, as they better capture essential local features.
\end{abstract}

\section{Introduction}

Times series data are commonly found in a variety of real-world applications, including financial data analysis, medical and health monitoring, industrial automation, image recognition, and so forth. Extracting useful knowledge from time series data - a.k.a. time series mining [1] - is now popular but remains a challenging research topic. In particular, time series classification (TSC) attracts significant interest among the researchers and industry practitioners. TSC approaches often implement supervised learning techniques to classify unknown time series instances based on knowledge gained from existing labeled ones.

Due to the abundance and intrinsic high dimensionality of time series data, computing resources such as storage, CPU and memory have become critical bottlenecks in the exploitation of time series. To address these challenges, the research community has proposed efficient dimensionality reduction [2] and representation mechanisms such as SAX [3] and Discrete Wavelet Transforms (DWT). DWT is very popular among both researchers and industry practitioners. In general, wavelets are mathematical functions that process raw data to only keep meaningful oscillations in data values. The earliest wavelet was brought up by Haar in 1909, but has undergone great development especially since Ingrid Daubechies [4] proved the existence of wavelet families with compact support over an interval [5] and orthogonal translates [6]. Wavelets compression techniques have since then been extensively used in image compression and are part of the JPEG 2000 standard [7]. Wavelet transforms have also been extensively used in medical data analysis including ECG diagnosis [8] and ultrasound image processing [9]. More recently, researchers have applied wavelet transforms in the field of Non-Intrusive Load Monitoring (NILM) [10, 11].

Although wavelets are popular in the research community, the literature lacks a large scale empirical study on the impact of wavelet transformation on the performance of time series mining approaches. In this paper, we seek to investigate this impact on a baseline state-of-the-art time series classification approach. To that end, we compare the classification accuracy of raw uncompressed time series data against transformed data using DWT, including both wavelet approximations and the details, i.e., the residuals or noises that are often thrown away during the lossy compression processes. In this way, we are able to separate time series' global features from local defining subsequences.

In this study, we extensively test how discrete wavelet transforms impact TSC accuracy and computational efficiency using 39 openly accessible datasets. Our study suggests that DWT can indeed be useful in time series classification tasks:

- Wavelet transforms can be used to reduce dimensionality of time series data, while at the same time achieving similar classification accuracy compared to using the original uncompressed data. In fact, we demonstrate that time series dimensionality may be reduced by around $90 \%$ while still achieving good classification accuracy.

- Wavelets can be used to reduce noises in time series data, so that better classification performance can be achieved after conducting wavelet transform on the original uncompressed data.

- We have further found that, surprisingly, for a few datasets, classification using the compression residual details can be even more effective than using either the original data or the wavelet approximation. This finding suggests that there are specific datasets which are more distinguishable using local features instead of global ones. 
The remainder of this paper is organized as follows. We introduce the necessary background information in Section 2 and related work in Section 3. We present experimental details and results in Section 4, before concluding the paper and outlining future work in Section 5.

\section{Background}

In this section, we present the necessary background to facilitate understanding of this paper. Specifically, we firstly introduce time series and the baseline classification approach used in this paper. Then we show how DWT works and especially how DWT can be applied to time series dimensionality reduction.

\subsection{Time Series and TSC}

Generally, time series is one important type of temporal data. In the data mining community, time series data are often referred to as ordered lists of numeric values [12]. In this paper, a time series $T=t_{0}, t_{1}, \ldots, t_{n-1}$, where $t_{i}(0 \leq$ $i \leq n-1)$ is a finite number and $T$ has a length of $n$, i.e., $|T|=n$.

Time series classification (TSC) is a common category of tasks that involves learning from existing time series instances (training set) and applying the learned knowledge to assign labels to instances from a testing dataset, where instance classes or labels are often unknown (either this information does not exist or has been intentionally hidden from the classification process). TSC tasks are especially common in application domains such as image and speech recognition (e.g., for recognizing spoken words), medical diagnosis (e.g., for detecting the type of a heart disease in an ECG signal), gesture detection, and so on. Due to its numerous application scenarios, many techniques have been proposed for TSC, including k-Nearest Neighbors, shapelets [13], and bag-of-features [14]. Among them, the Nearest Neighbor (1NN) approach has been proven to work exceptionally well, especially when using Dynamic Time Warping (DTW) [15] for computing the distance metric between a pair of time series samples. Figure 1 shows an example of how DTW works. Unlike Euclidean distance where two time series are aligned point by point, i.e., the $i^{t h}$ point in series $\mathrm{X}$ is compared against the $i^{t h}$ point in another series Y, DTW tries to find the best way to warp the time axis and as a result aligns $\mathrm{X}$ and $\mathrm{Y}$ differently. As shown by the gray dotted lines in Figure 1 , an $i^{t h}$ point in $\mathrm{X}$ can be mapped to a $j^{\text {th }}$ point (it is possible that $i \neq j$ ), or one point in X may even be mapped to multiple points in Y. For Euclidean distance, the gray dotted lines would all be vertical. Thanks to DTW's capability to describe similarities, we consider DTW-based $1 \mathrm{NN}$ classification as a reference TSC approach for investigating wavelet transformed time series data.

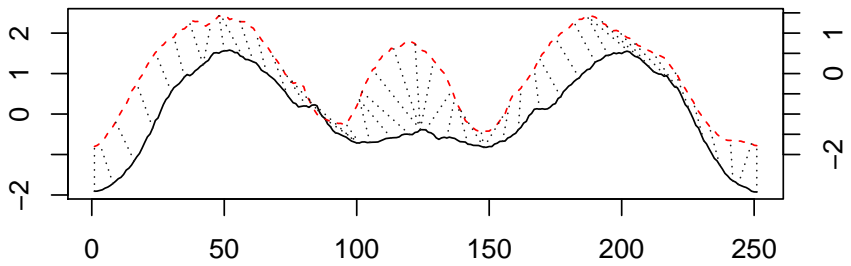

Figure 1: Illustration of how DTW aligns two time series and calculates their distance.

\subsection{Discrete Wavelet Transforms}

Wavelets are mathematical functions that resemble the shape of wave oscillations, with the constraint for waves to start at 0 and then oscillate to 0 in the end. Mathematically, wavelets are described using two types of functions: the wavelet function (the mother function denoted with $\psi(t)$ ) and the scaling function (the father function denoted with $\phi(t))$ [16]. These functions have to satisfy the following conditions:

$$
\begin{gathered}
\|\psi(t)\|^{2}=\int|\psi(t)|^{2} d t<\infty \\
\int|\psi(t)| d t<\infty, \int \psi(t) d t=0, \int \phi(t) d t=1
\end{gathered}
$$

The earliest and simplest wavelet function is Haar, whose wavelet function and scaling function are defined as follows:

$$
\begin{gathered}
\psi_{\text {Haar }}(t)= \begin{cases}-1 & 1 / 2 \leq t<1 \\
1 & 0 \leq t<1 / 2 \\
0 & \text { otherwise }\end{cases} \\
\phi_{\text {Haar }}(t)= \begin{cases}1 & 0<t<1 \\
0 & \text { otherwise }\end{cases}
\end{gathered}
$$

As illustrated in Figure 2, the Haar wavelet is not a very smooth one, that is, it has a low regularity. Other more sophisticated wavelets - for instance, Daubechies 20 and Symlets 20 - have a higher regularity and thus are able to represent signals more accurately.
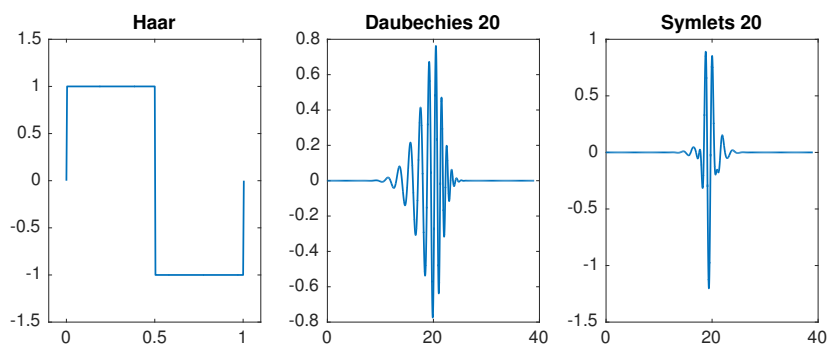

Figure 2: Wavelet functions of Haar, Daubechies 20 and Symlets 20.

In practice, the Haar transform is performed as follows. Given a series $T=t_{1}, \ldots, t_{n}$, the Haar transform outputs two series: the approximation $A$ and the details $D$, where for $1 \leq i \leq \frac{n}{2}$, 


$$
A_{i}=\frac{t_{2 i-1}+t_{2 i}}{\sqrt{2}}, D_{i}=\frac{t_{2 i-1}-t_{2 i}}{\sqrt{2}}
$$

It is clear that the approximations capture the overall shape - the global features - of the original series, while the details are the variances - local features - in time series. Figure 3 demonstrates an example of single level, one dimensional Haar transform. As shown, the original signal on the top has been compressed to half of the original size (figure in the middle, note the $\mathrm{x}$-axis label) and the amplitude has been scaled up despite that the overall signal shape is not changed. Note also the grayed area in the signal, it is clear that the approximation becomes smoother after transformation, and that the details shown in the bottom have been dropped.

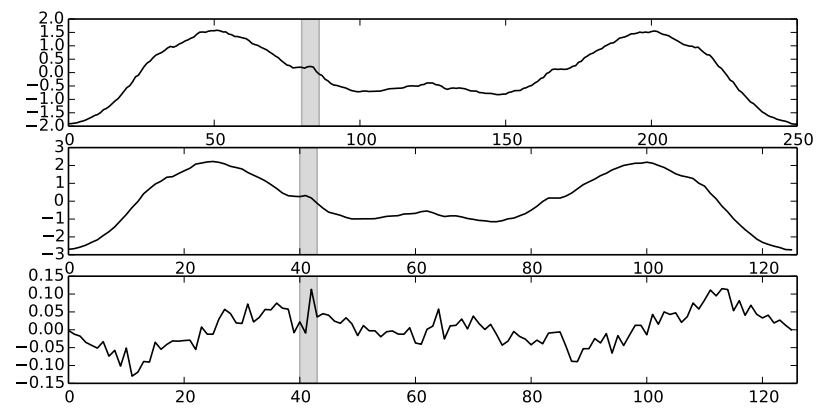

Figure 3: Example of Haar transform: the original signal, the Haar approximation and the residual details.

\section{Related Work}

Thanks to wavelets' wide application domains [17], there has been research investigating their performance in various domains. However, to the best of our knowledge, impact of different DWT techniques on TSC has not been done in a generic manner. As a result, in this section we enumerate relevant work to ours in the domains of medical applications, image compression, NILM, and so on.

Addison [8] has conducted a review of both continuous wavelet transform (CWT) and discrete wavelet transform (DWT) on ECG data, concluding that DWT is practically easy to use, while CWT - being more complex and difficult to tune parameters - is able to keep a high resolution in the time-frequency plane, which can result in more accurate identification of components. Another study in wavelet applications in the medical field was done by Pizurica et. al. [9], where the authors reviewed the performance of wavelet denoising specifically in MRI and brain imaging.

To disaggregate electric signals, Duarte et. al. [10] take advantage of CWT in order to extract features from voltage transients and use the extracted features for classification. Gray et. al. [11] use wavelet-based classification for NILM and claim "symlets behave in an identical manner as their less symmetric Daubechies representation". But as our empirical study shall demonstrate in the next section, Symlets actually perform better than Daubechies.

Chan et. al. [18] have proposed using Haar for more efficient similarity search, but have not considered other wavelet families. Finally, this work was partly inspired by our previous work, where we have taken advantage of SAX to transform/compress time series data and then build perclass language models $[19,20]$. When classifying, we compare time series against models instead of known samples.

\section{Experimental Study}

In this section, we present our experimental setup and the collected results. In order to facilitate reproducibility, we opt to experiment on publicly available datasets, and further open source our own implementation ${ }^{1}$.

Table 1: Characteristics of datasets used in our study.

\begin{tabular}{llcccc}
\hline \# & Dataset Name & \#Classes & \#Training & \#Testing & Length \\
\hline 1 & ArrowHead & 3 & 36 & 175 & 251 \\
2 & BeetleFly & 2 & 20 & 20 & 512 \\
3 & BirdChicken & 2 & 20 & 20 & 512 \\
4 & Computers & 2 & 250 & 250 & 720 \\
5 & DistalPhalanxOutlineAgeGroup & 3 & 139 & 400 & 80 \\
6 & DistalPhalanxOutlineCorrect & 2 & 276 & 600 & 80 \\
7 & DistalPhalanxTW & 6 & 139 & 400 & 80 \\
8 & Earthquakes & 2 & 139 & 322 & 512 \\
9 & ECG5000 & 5 & 500 & 4500 & 140 \\
10 & ElectricDevices & 7 & 8926 & 7711 & 96 \\
11 & FordA & 2 & 1320 & 3601 & 500 \\
12 & FordB & 2 & 810 & 3636 & 500 \\
13 & Ham & 2 & 109 & 105 & 431 \\
14 & HandOutlines & 2 & 370 & 1000 & 2709 \\
15 & Herring & 2 & 64 & 64 & 512 \\
16 & InsectWingbeatSound & 11 & 220 & 1980 & 256 \\
17 & LargeKitchenAppliances & 3 & 375 & 375 & 720 \\
18 & Meat & 3 & 60 & 60 & 448 \\
19 & MiddlePhalanxOutlineAgeGroup & 3 & 154 & 400 & 80 \\
20 & MiddlePhalanxOutlineCorrect & 2 & 291 & 600 & 80 \\
21 & MiddlePhalanxTW & 6 & 154 & 399 & 80 \\
22 & PhalangesOutlinesCorrect & 2 & 1800 & 858 & 80 \\
23 & Phoneme(readme) & 39 & 214 & 1896 & 1024 \\
24 & ProximalPhalanxOutlineAgeGroup & 3 & 400 & 205 & 80 \\
25 & ProximalPhalanxOutlineCorrect & 2 & 600 & 291 & 80 \\
26 & ProximalPhalanxTW & 6 & 205 & 400 & 80 \\
27 & RefrigerationDevices & 3 & 375 & 375 & 720 \\
28 & ScreenType & 3 & 375 & 375 & 720 \\
29 & ShapeletSim & 2 & 20 & 180 & 500 \\
30 & ShapesAll & 60 & 600 & 600 & 512 \\
31 & SmallKitchenAppliances & 3 & 375 & 375 & 720 \\
32 & Strawberry & 2 & 370 & 613 & 235 \\
33 & ToeSegmentation1 & 2 & 40 & 228 & 277 \\
34 & ToeSegmentation2 & 2 & 36 & 130 & 343 \\
35 & UWaveGestureLibraryAll & 8 & 896 & 3582 & 945 \\
36 & Wine & 2 & 57 & 54 & 234 \\
37 & WordSynonyms & 25 & 267 & 638 & 270 \\
38 & Worms & 5 & 77 & 181 & 900 \\
39 & WormsTwoClass & 2 & 77 & 181 & 900 \\
\hline & Setup and Datas & & & &
\end{tabular}

\subsection{Setup and Datasets}

The goal of our study is to investigate how wavelet transformed data may impact TSC performance. Therefore, we do not intend to compare the performance of different classifiers and similarity metrics (which have been empirically studied in [21]). We rely in our study on the most frequently used classification method: Nearest Neighbor Classification (1NN) with DTW distance. In this paper we choose to calculate the classification performance using FastDTW [22],

\footnotetext{
${ }^{1}$ https://github.com/serval-snt-uni-lu/wavelets-tsc
} 


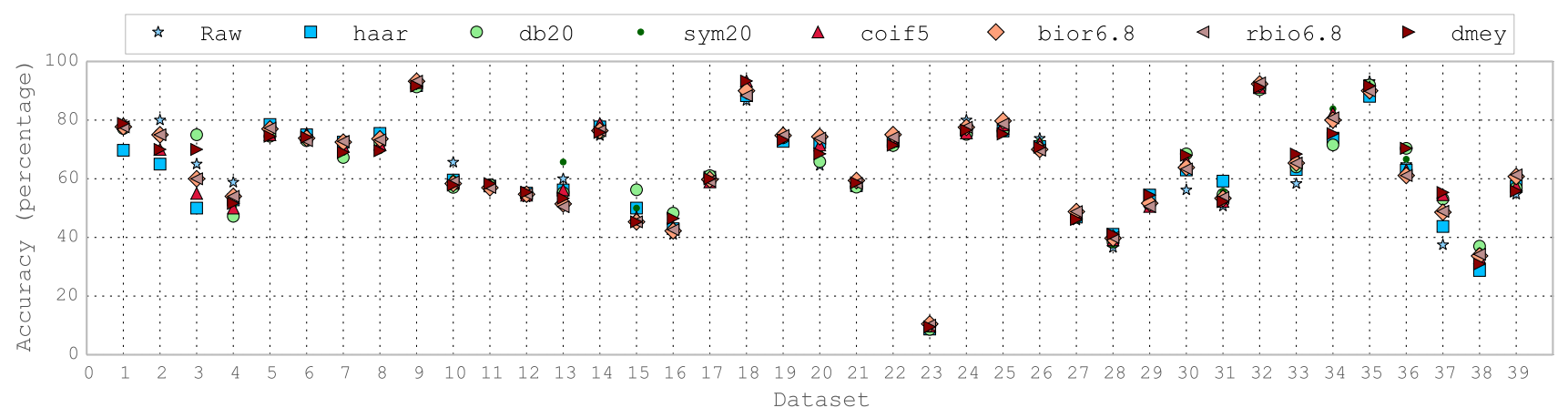

Figure 4: Classification accuracy with FastDTW based 1NN, using original and DWT transformed/compressed data.

which is a DTW approximation with linear time complexity. Although we are more familiar with FastDTW, please note that the UCR Suite [23] is exact instead of approximated and it can be faster than FastDTW.

The datasets that we have experimented on are from the UCR Time Series Classification Archive [24], which contains datasets from various domains ranging from electricity readings and medical signals such as Electrocardiographs (ECGs) to image recognition data. We have specifically chosen to use the NewlyAddedDatasets, which contains 39 separate datasets from various domains, with all these datasets sharing a unified file format and internal representation structure, which is convenient for batch processing. The UCR archive provides both the datasets and the ground-truth, i.e., the correct label of each testing instance.Table 1 summarizes the characteristics of these 39 datasets. As shown, this archive comes with predefined training and testing sets, which makes it easier for researchers to compare results in an uniformed manner. Besides, it is obvious that several datasets are large in size, for instance, to classify all 7,711 testing instances using $1 \mathrm{NN}$ in ElectricDevices (\#10), there will be $7,711 * 8,926=68,828,386$ pairwise comparisons, making the classification process extremely time consuming.

\subsection{TSC with Wavelet Transformed Data}

As a first step, we seek to investigate how DWT compressed data will impact classification accuracy compared with using raw uncompressed data. Here we transform all the 39 datasets using wavelets from seven well-known families, choosing the single wavelet with the highest regularity from each family. Concretely, the wavelets are: Haar, Daubechies 20, Symlets 20, Coiflets 5, Biorthogonal 6.8, Reverse biorthogonal 6.8 and Discrete Meyer with finite impulse response (FIR) approximation. In this step, all time series - both training instances and testing instances - from each dataset are processed using single level, one dimensional DWT. After transformation, the size of compressed data is reduced by half from the original series. Then, we use FastDTW-based 1NN to classify all the testing instances in each dataset. Thanks to the $O(n)$ time complexity of
FastDTW, reducing time series sizes by half means reducing classification time by half. And for DTW with $O\left(n^{2}\right)$ complexity, classification time can be reduced by $75 \%$.

Figure 4 presents the classification accuracy of each wavelet transformed dataset together with that of the original data. Accuracies highlighted in bold indicate equivalent or better performance using wavelet transformed data. We note that, while the compression yields smaller datasets and leads to faster classification, it does not impact the classification accuracy in most cases. Furthermore, in the case of several datasets, the classification accuracy has actually been improved on compressed data. These observations suggest that wavelet transformations are indeed relevant means for noise reduction in TSC tasks.

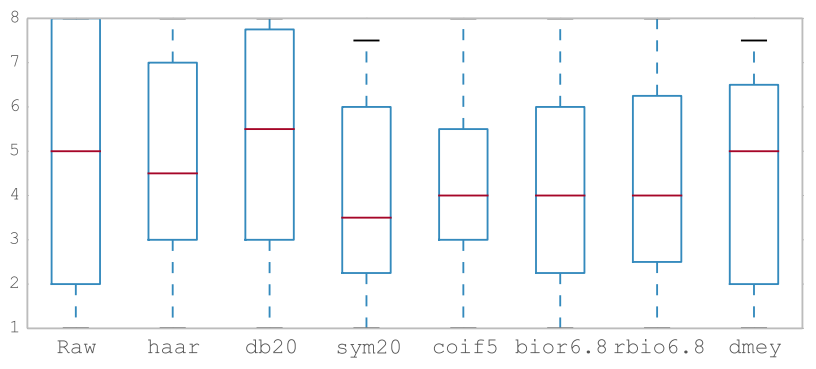

Figure 5: Rank of classification accuracy by approximation of different wavelet transformation.

To further investigate which wavelet family performs better globally, we rank each wavelet family's classification performance per dataset and draw a boxplot chart of these rankings. As shown in Figure 5, in general wavelet transform data performs better compared with the original data, thanks to wavelets' noise reduction functionality. Regarding the performance of individual wavelets, Symlets 20 generally outperforms the rest, including classification using original data. An much to our surprise, Daubechies 20 in overall performs the worst among all tested wavelets, indicating that the most smooth wavelet may not be the most suitable wavelet for TSC.

\subsection{TSC with Residual Details}

It is intuitive that when using wavelet transformation, the approximation of original data keeps more relevant infor- 


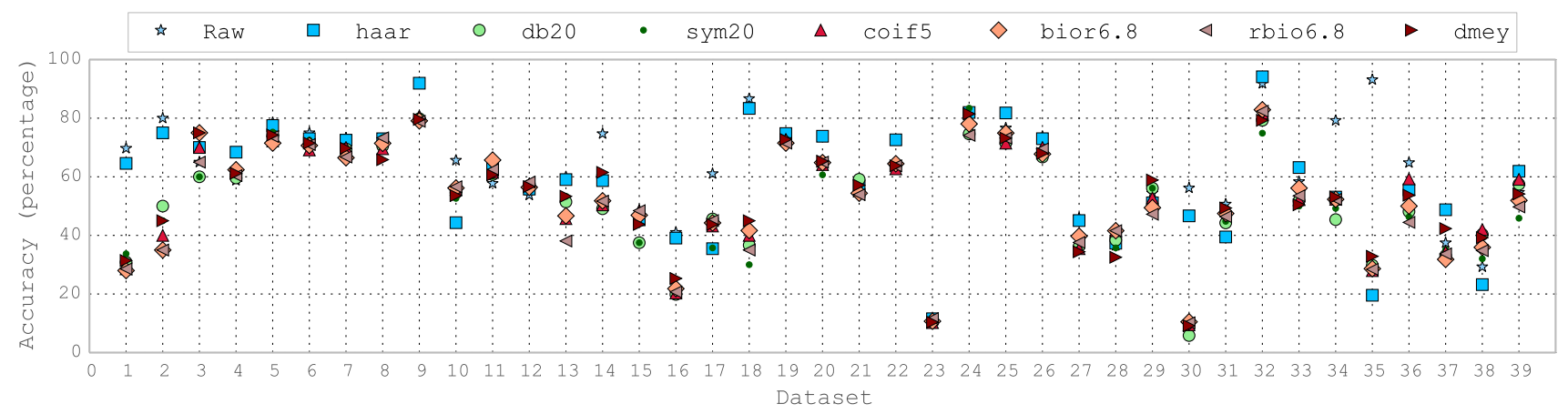

Figure 6: Classification accuracy with FastDTW based 1NN, using original data and residual details from DWT transform.

mation than the residual details. However, in the next experiments, we demonstrate that the residual details may be also useful for TSC and that in some scenarios the noises are more discriminative features than the approximations. To prove this seemingly counter-intuitive point, we follow the same procedures as Section 4.2 to compress all datasets, while instead of keeping the approximations, here we drop all of them and consider the residual details, i.e., the noises. Again, to be fair, we compare the classification accuracies using the noises against that using the original untransformed data.

Figure 6 presents the classification accuracies using only the residual details. As shown, although the classification accuracies are generally lower when classifying using only the residuals, they are still surprisingly high in many cases. Especially, for several datasets, classification accuracy using only residuals are similar or higher than using the raw data. Next, we try to rank how discriminative the residuals from each wavelet family are. The boxplot shown in Figure 7 suggests that residuals produced by Haar are most discriminative, being almost as good as the original data. Symlets 20, on the other hand, falls on the other extreme. These two observations are in accordance with the finding in Section 4.2, suggesting that Symlets 20 is best at keeping globally relevant information during transformation. This observation suggests that residuals from DWT compression can also be useful, since these details contain local features that are potentially discriminative.

As a result, we believe that both the approximation and details transformed from the original data are important, due to the fact that the transforms extract two independent discriminative features. While some datasets are more distinguishable using global features, others are more so using local defining features.

\subsection{Multi-Level Wavelet Transformation}

So far we have only tested the performance of single level wavelet transformation. Since it is possible to conduct wavelet transformation in multiple levels, we seek to investigate how TSC performance can be affected when transforming data through multiple levels. To be specific, we

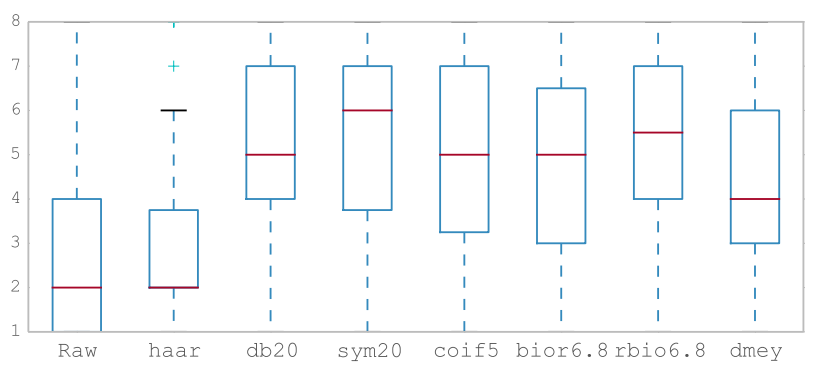

Figure 7: Rank of classification accuracy by residual details from different wavelet transformation.

transform all datasets with Symlets 20 from level 2 till the maximum level. That is, due to different time series lengths, even level 2 transformation is not possible for short time series, while longer time series may be processed using level 6 Symlets 20 decomposition. In Table 2 we present both the percentage of dimension/size reduction $(\mathbf{R})$ and the corresponding classification accuracy $(\mathbf{A})$ in each level. Note that not all datasets support at least level 2 decomposition due to short lengths, as a result, these datasets are omitted in this table.

As shown in Table 2, although many datasets are compressed by $80 \%$ to $90 \%$ in size, the classification accuracy using these reduced data can still outperform those using original compressed data. As a result, we think it is safe to claim that multi level wavelet transformation are indeed helpful for TSC tasks when it comes to classifying long time series. Note especially the HandOut lines (\#14) dataset, due to its extremely high dimensionality, we are able to compress them by up to $97 \%$ of the original size, while still received remarkably high classification accuracy. Since FastDWT normally has a time complexity of $O(n)$, this indicates huge time savings in the classification process.

\section{Conclusions and Future Work}

Discrete Wavelet Transform techniques have matured in the past decades to deliver high data compression rates. Applied to time series data, existing DWT-based lossy compression approaches help to overcome the challenges of storage and computation time. In this paper, we provide assurances to practitioners by empirically showing with var- 
Table 2: Classification accuracy with FastDTW based 1NN, using Symlets 20 multi-level decomposition.

\begin{tabular}{|c|c|c|c|c|c|c|c|c|c|c|c|}
\hline \multirow[b]{2}{*}{ \# } & \multirow[t]{2}{*}{ Raw } & \multicolumn{2}{|c|}{ Level 2} & \multicolumn{2}{|c|}{ Level 3} & \multicolumn{2}{|c|}{ Level 4} & \multicolumn{2}{|c|}{ Level 5} & \multicolumn{2}{|c|}{ Level 6} \\
\hline & & $\mathbf{R}$ & $\mathbf{A}$ & $\mathbf{R}$ & $\mathbf{A}$ & $\mathbf{R}$ & $\mathbf{A}$ & $\mathbf{R}$ & $\mathbf{A}$ & $\mathbf{R}$ & $\mathbf{A}$ \\
\hline 1 & 69.7 & 63.3 & 78.3 & & & & & & & & \\
\hline 2 & 80.0 & 69.3 & 65.0 & 80.9 & 65.0 & & & & & & \\
\hline 3 & 65.0 & 69.3 & 70.0 & 80.9 & 80.0 & & & & & & \\
\hline 4 & 58.8 & 71.0 & 58.4 & 82.8 & 55.2 & 88.8 & 50.0 & & & & \\
\hline 8 & 71.7 & 69.3 & 78.6 & 80.9 & 69.9 & & & & & & \\
\hline 11 & 57.7 & 69.2 & 56.5 & 80.8 & 59.7 & & & & & & \\
\hline 12 & 53.8 & 69.2 & 55.9 & 80.8 & 62.0 & & & & & & \\
\hline 13 & 60.0 & 68.2 & 65.7 & 79.6 & 53.3 & & & & & & \\
\hline 14 & 74.7 & 73.9 & 80.0 & 86.3 & 79.9 & 92.4 & 79.0 & 95.5 & 71.1 & 97.0 & 70.7 \\
\hline 15 & 45.3 & 69.3 & 53.1 & 80.9 & 50.0 & & & & & & \\
\hline 16 & 41.0 & 63.7 & 44.1 & & & & & & & & \\
\hline 17 & 61.1 & 71.0 & 64.0 & 82.8 & 63.2 & 88.8 & 64.3 & & & & \\
\hline 18 & 86.7 & 68.5 & 93.3 & 79.9 & 93.3 & & & & & & \\
\hline 23 & 9.9 & 72.2 & 12.3 & 84.2 & 12.4 & 90.2 & 11.0 & & & & \\
\hline 27 & 45.9 & 71.0 & 45.3 & 82.8 & 41.9 & 88.8 & 39.2 & & & & \\
\hline 28 & 36.5 & 71.0 & 36.8 & 82.8 & 38.4 & 88.8 & 40.0 & & & & \\
\hline 29 & 54.4 & 69.2 & 55.0 & 80.8 & 51.7 & & & & & & \\
\hline 30 & 56.2 & 69.3 & 72.7 & 80.9 & 72.2 & & & & & & \\
\hline 31 & 50.7 & 71.0 & 61.9 & 82.8 & 65.9 & 88.8 & 67.5 & & & & \\
\hline 32 & 91.8 & 62.6 & 91.7 & & & & & & & & \\
\hline 33 & 58.3 & 64.6 & 68.0 & & & & & & & & \\
\hline 34 & 79.2 & 66.5 & 81.5 & 77.6 & 77.7 & & & & & & \\
\hline 35 & 93.1 & 72.0 & 92.4 & 83.9 & 93.2 & 89.9 & 91.5 & & & & \\
\hline 36 & 64.8 & 62.8 & 55.6 & & & & & & & & \\
\hline 37 & 37.5 & 64.4 & 61.3 & & & & & & & & \\
\hline 38 & 29.3 & 71.8 & 35.9 & 83.8 & 45.3 & 89.8 & 47.0 & & & & \\
\hline 39 & 54.7 & 71.8 & 60.2 & 83.8 & 64.6 & 89.8 & 65.7 & & & & \\
\hline
\end{tabular}

ious datasets and with several DWT approaches that time series classification yields similar accuracy on both compressed (i.e., approximated) and raw time series data. We also show that, in some datasets, wavelets may actually help in reducing noisy variations which deteriorate the performance of mining tasks. In a few cases, we note that the residual details/noises from compression are more useful for recognizing data patterns.

In future work, we plan to extensively investigate the characteristics of time series datasets, in order to empirically correlate successful wavelets techniques per application domains. Dataset characterizations will also help identify which types of time series data can benefit from the use of residual details instead of the approximation data.

\section{References}

[1] T.-C. Fu, "A review on time series data mining," Engineering Applications of Artificial Intelligence, vol. 24, no. 1, pp. 164-181, 2011.

[2] Q. Wang and V. Megalooikonomou, "A dimensionality reduction technique for efficient time series similarity analysis," Information systems, vol. 33, no. 1, pp. 115-132, 2008.

[3] J. Lin, E. Keogh, L. Wei, and S. Lonardi, "Experiencing sax: a novel symbolic representation of time series," Data Mining and knowledge discovery, vol. 15, no. 2, pp. 107-144, 2007.

[4] I. Daubechies, "Orthonormal bases of compactly supported wavelets," Communications on pure and applied mathematics, vol. 41, no. 7, pp. 909-996, 1988.

[5] A. Cohen, I. Daubechies, and P. Vial, "Wavelets on the interval and fast wavelet transforms," Applied and computational harmonic analysis, vol. 1, no. 1, pp. 54-81, 1993.

[6] I. Daubechies, "Orthonormal bases of compactly supported wavelets ii. variations on a theme," SIAM Journal on Mathematical Analysis, vol. 24, no. 2, pp. 499-519, 1993.

[7] D. S. Taubman and M. W. Marcellin, "JPEG2000: Standard for interactive imaging," Proceedings of the IEEE, vol. 90, no. 8, pp. 13361357, 2002.
[8] P. S. Addison, "Wavelet transforms and the ECG: a review," Physiological measurement, vol. 26, no. 5, p. R155, 2005.

[9] A. Pizurica, A. M. Wink, E. Vansteenkiste, W. Philips, and B. J. Roerdink, "A review of wavelet denoising in MRI and ultrasound brain imaging," Current medical imaging reviews, vol. 2, no. 2, pp. 247-260, 2006

[10] C. Duarte, P. Delmar, K. W. Goossen, K. Barner, and E. GomezLuna, "Non-intrusive load monitoring based on switching voltage transients and wavelet transforms," in Future of Instrumentation International Workshop (FIIW), 2012. IEEE, 2012, pp. 1-4.

[11] M. Gray and W. Morsi, "Application of wavelet-based classification in non-intrusive load monitoring," in Electrical and Computer Engineering (CCECE), 2015 IEEE 28th Canadian Conference on. IEEE, 2015, pp. 41-45.

[12] T. Rakthanmanon and E. Keogh, "Fast shapelets: A scalable algorithm for discovering time series shapelets," in Proceedings of the thirteenth SIAM conference on data mining, 2013.

[13] L. Ye and E. Keogh, "Time series shapelets: a new primitive for data mining," in Proceedings of the 15th ACM SIGKDD international conference on Knowledge discovery and data mining. ACM, 2009, pp. 947-956.

[14] M. G. Baydogan, G. Runger, and E. Tuv, "A bag-of-features framework to classify time series," IEEE Transactions on Pattern Analysis and Machine Intelligence, vol. 35, no. 11, pp. 2796-2802, 2013.

[15] G. E. Batista, X. Wang, and E. J. Keogh, "A complexity-invariant distance measure for time series." in SDM, vol. 11, 2011, pp. 699710.

[16] K. Amolins, Y. Zhang, and P. Dare, "Wavelet based image fusion techniques - An introduction, review and comparison," ISPRS Journal of Photogrammetry and Remote Sensing, vol. 62, no. 4, pp. 249263, 2007.

[17] A. K.-m. Leung, F.-t. Chau, and J.-b. Gao, "A review on applications of wavelet transform techniques in chemical analysis: 1989-1997,' Chemometrics and Intelligent Laboratory Systems, vol. 43, no. 1, pp. 165-184, 1998

[18] F. K.-P. Chan, A. W.-c. Fu, and C. Yu, "Haar wavelets for efficient similarity search of time-series: with and without time warping," Knowledge and Data Engineering, IEEE Transactions on, vol. 15, no. 3, pp. 686-705, 2003.

[19] D. Li, T. F. Bissyande, S. Kubler, J. Klein, and Y. Le Traon, "Profiling Household Appliance Electricity Usage with N-Gram Language Modeling," in The 2016 IEEE International Conference on Industrial Technology (ICIT 2016). Taipei: IEEE, 2016, pp. 604-609.

[20] D. Li, L. Li, T. F. Bissyande, J. Klein, and Y. Le Traon, "DSCo: A Language Modeling Approach for Time Series Classification," in The 12th International Conference on Machine Learning and Data Mining (MLDM 2016). New York: Springer, 2016.

[21] J. Serrà and J. L. Arcos, "An empirical evaluation of similarity measures for time series classification," Knowledge-Based Systems, vol. 67, pp. 305-314, 2014.

[22] S. Salvador and P. Chan, "Toward accurate dynamic time warping in linear time and space," Intelligent Data Analysis, vol. 11, no. 5, pp. 561-580, 2007.

[23] T. Rakthanmanon, B. Campana, A. Mueen, G. Batista, B. Westover, Q. Zhu, J. Zakaria, and E. Keogh, "Searching and mining trillions of time series subsequences under dynamic time warping," in Proceedings of the 18th ACM SIGKDD international conference on Knowledge discovery and data mining. ACM, 2012, pp. 262-270.

[24] Y. Chen, E. Keogh, B. Hu, N. Begum, A. Bagnall, A. Mueen, and G. Batista, "The ucr time series classification archive," July 2015, www.cs.ucr.edu/ eamonn/time_series_data/. 